\title{
The Structural Complexity of Various Elements of Systems
}

\author{
Tomi Tristono*, Fardiana Karuniawati, Setiyo Daru Cahyono, \\ Seno Aji and Pradityo Utomo
}

\author{
Faculty of Engineering, University of Merdeka Madiun, East Java 63133, Indonesia
}

('Corresponding author's e-mail: tomitristono@gmail.com, tomitristono@unmer-madiun.ac.id)

Received: 22 September 2020 Revised: 27 April 2021 Accepted: 22 May 2021

\begin{abstract}
This paper is aimed to describe the structural complexity of the traffic light control system. The structural complexity represents an interrelationship indicator between the various elements functions. The traffic light control system has several additional features that are synchronized to the railway doorstop, and the time interval for the green signals is expanded. For determining the method of its structural complexity, the Petri net model is used. Three kinds of traffic lights models are explored, namely standard, Norwegian, and the Norwegian improvement. The study results indicate that the Norwegian traffic lights have the most complex structure when regular implementation. The runner up is the Norwegian improvement. The most ideal is the standard traffic lights. Norwegian improvement is the most ideal when integrated with the railway doorstop and implemented extra green signals time interval. The Norwegian traffic light is the second. The standard traffic lights structural complexity fluctuates. It means that the Norwegian traffic light and its improvement are suitably used. Adding several features improve the structural complexity approaching the ideal system while extra controllers accompany each element.
\end{abstract}

Keywords: Control system, Petri net, Structural complexity, Traffic lights, Various elements

\section{Introduction}

The structural complexity indicates the interrelationship of the various elements of a system. In general, system complexity consists of scope, uncertainty, and dynamics. A simple design that is not complex does not involve the dynamics and suspense of a state. Improvements in the functions of the devices always increase system complexity. An elevate of coordination and control functions is coupled with expensive and sophisticated methods [1]. The structural complexity learns about coordination ability concerning the coordinator's function and its relation to the global task [2]. The structural complexity is the constructional complexity. It is different from computational complexity that discusses the time and memory space required by a computational process [3].

This study is aimed to determine the structural complexity of the traffic lights control system as a feasibility test for actual implementation. It studies the structural complexity of the single-phase, 2-phase, 3-phase, and 4-phase traffic lights control system. They also have dual features; both are synchronized to the railway doorstop and expanded its green signals time interval [4]. Several control system kinds which we discussed are standard, Norwegian traffic lights, and the Norwegian improvement.

For determining methods of structural complexity, the Petri net model is used. Petri net represents a Discrete Event System (DES) that can use to model the structure of the traffic lights control systems. The traffic lights states are connected with the specific time interval of the occurrence of events. Typically, DES involves a relationship with the environment, conflict of resources, and simultaneous occurrence activities [5]. Petri nets with extensions increase the modularity of the control systems and describe the actual-time constraints [6]. Petri net is the mathematical design using graphics and formal method for massive size system, which attaches concurrency, dynamic behaviour, synchronous or asynchronous communication, and resource sharing [7].

In the following have been written the previous researches. The study determined the simulation complexity of a model. It implemented DES. It was the research of Popovicsa and Monostoria [8]. The Maraghy et al. [9] had analyzed the system complexity of engineering design and manufacturing [9]. Hariri and Perkasa had studied e-learning system complexity. They used function-oriented metrics [10]. Taiping and Peisi applied Petri net to analyze the manufacturing system [11]. Dong and Li [12] had 
employed the fuzzy Petri-net is to investigate the system fault diagnosis. Mukhlash et al. [13] had used the coloured Petri nets to improve the business process of the production systems. Ouelhadj and Petrovic [14] had reviewed the dynamic schedule of the manufacturing system. Han and Tian [15] have used the Petri net model to improve conformance checking of business process management. The real-time measurements for the responsive traffic signals. It was M Soares's research [16]. Yang and Xiao-bo [17] collaborated the fuzzy Petri net with the genetic algorithm. Hartanti et al. [18] built a smart traffic light to prevent traffic congestion. Kurniawan et al. [19] had analyzed the adaptive traffic lights controller problem. They used Pre-Timed System. Zinovyev and Mirkesde [20] had used the principal graphs to calculate data complexity. Zenil et al. [21] had studied the graph and network complexity based on an algorithmic information perspective. Neel and Orrison [22] had studied the linear complexity of a graph. Basir [23] has analyzed the growth of space and time complexity on the heap sort, insertion sort and merge algorithm. Java programming is used. Lee and Chiu [24] have designed a smart traffic signal control system implemented by smart city applications.

Many researchers have investigated the system complexity. None of their research is associated with the structural complexity of the traffic lights control systems. This method contributes the authorities to choose the feasible design for actual use. Advanced systems that have a high level of complexity is usually complicated and expensive. It will not cause problems when the budget is widely available, and there are many experts. Add features will be beneficial for road users. Conversely, while the budget is limited and the number of experts is a few, you should use a simple system.

\section{Materials and methods}

The traffic light model built using the classic Petri net. For the validation and verification of the model, it used Petri net Simulator 2.0. It must show that the model is correct and satisfies all the Petri net properties.

\section{Design of traffic lights control system using Petri net}

Several elements that are part of the Petri net include places, transitions, arcs and tokens. Places (circles) indicate the states. The transitions (rectangles) task is to trigger the sequence of states. The transitions represent the event that occurred. The arcs represent the direction of the state's stream. If tokens exist in a place, it is a mark that the related state is active. It is termed marking [25]. In this paper, marking $=\{0,1\}=$ turn off, turn on $\}$.

The pre-set and post-set of the transitions are all input places and output places of a transition. ${ }^{*} t$ terms of the input places and $t^{\bullet}$ represents the output places. The pre-set and post-set of places are the input transitions and output transitions of a place. They are ${ }^{\bullet} p$ and $p^{\bullet}$, respectively [25].

The places of the 2-phase traffic lights control system are GS (Green South), YS (Yellow South), RS (Red South), GE (Green East), YE (Yellow East) and RE (Red East). All places other than signals are the control systems. The intermediation places, i.e., S1 and S2, play a role to create a traffic light phase order. Both states of S1 and S2 unvisualized like the traffic signal states. All traffic signals of an intersection turn on a red light while a token exists in S1 or S2. It is the pause time interval for emptying an intersection of the vehicles that unfinished passing. It is for travel safety reason. It intends to prevent vehicle traffic from conflict with flows from different directions [26]. An illustration of the model is in Figure 1.

The railway doorstop states are OP (Open) and CO (Closed). It may not transform RS (Red South) state into GS (Green South) if the railway doorstop is closed.

A place GS (Green South) expanded into 4 places for the time interval refinement. The time intervals: 1) GSmin (Green South minimum) 2) GS1 (Green South attached with the extra time 4 seconds) 3) GS2 (Green South which added time 8 s) and 4) GSmax (Green South maximum that has extra time $12 \mathrm{~s}$ ). The "traffic evaluation" done while $4 \mathrm{~s}$ before GSmin, GS1 and GS2 end. If any passing vehicles detected while the first $3 \mathrm{~s}$ when "traffic evaluation," the controller instructs for extra green signals when the last second of "traffic evaluation". Conversely, if undetected vehicles pass while the first 3 s when "traffic evaluation," the controller informs the green signal must terminate even though it has not yet reached the maximum time interval. The vehicle detectors gather the actual traffic watching. All detectors model is not attached. 


\section{Structural complexity based on the Petri net model}

The model complexity strongly connected to the characteristics of the complexity of the existing control system. The elements and structural complexity of the model reflect its static characteristics. Meaning, the states and their transformation process describe the dynamic behaviour of the system [14].
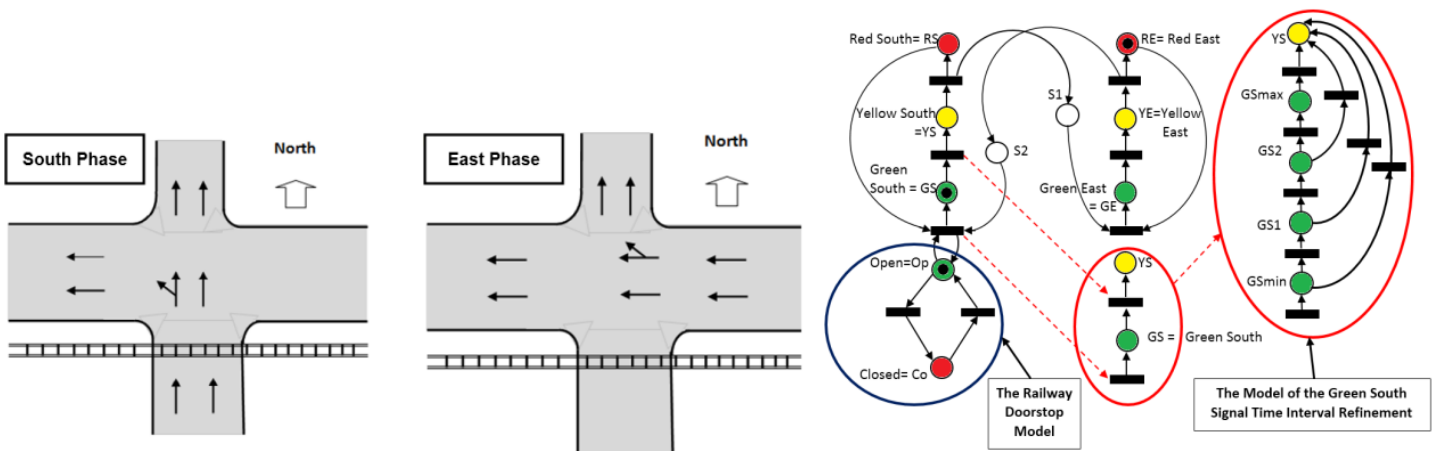

Figure 1 The traffic flow of movement a 2-phase traffic lights and the Petri net model.

It refers to Figure 1. The traffic movement of the south-phase is on the left side. The vehicle's traffic of east-phase is in the centre. Arrowheads represent the traffic movement. The railway intersects the south arm of the signalized intersection. The traffic lights carry out the eastern phase only when the train is passing. The green signal's time interval setting of the east arm is longer than the regular schedule. The system does it when the railway doorstop closes until the train finishes passing. The traffic lights Petri net model is put on the right side. The integrated model with the railway doorstop is inside the red ovoid in the model refinement of GS (Green South). Both traffic that comes from the east arm and south arm is 1-way movement entering the intersection. The vehicle's traffic of both the west arm and north arm keep away from the road intersection.

The terminology of the traffic lights structural complexity in this paper is as follows. $C_{n}$ symbolizes the elements complexity, and $C_{r}$ represents the average of the places and transitions correlation complexity. $C_{s}$ indicates structural complexity. The sum of $C_{n}$ and $C_{r}$ using the coefficient of the correlation $\lambda$ yields $C_{s}$ [15]. The incoming and outgoing arcs ratio to the entire connecting arcs of an element is the correlation coefficient. $|\mathrm{F}|$ is the number of arcs in the model.

$\lambda=\frac{1}{|T|+|P|} \sum_{i=1}^{|T|+|P|}\left(\frac{\left.\right|^{\bullet} x_{i}|+| x_{i}^{*} \mid}{|X|}\right)$ which $x_{i} \in X$

In Eq. (1), $|X|$ is the elements of the system. They should have pre-set and post-set. Both pre-set and post-set are not allowed to be empty. All elements in the model of the traffic lights control system are always have pre-set and post-set. None of them is empty. Why does this happen? It because the traffic lights behaviour is must repeat in cycles.

$C_{n}=\frac{1}{2}\left(\frac{|P|-1}{|P|}+\frac{|T|-1}{|T|}\right)$

The places correlation complexity of the system, the formula is written in Eq. (3) [15]. $|P|=\mathrm{m}$.

$C_{P}=\frac{1}{|P|} \sum_{i=1}^{|P|}\left(\frac{\left|p_{i}^{\bullet}\right|}{\left|\cdot p_{i}\right|+\left|p_{i}^{\bullet}\right|}\right)$

The transitions correlation complexity of the model, the formula is described in Eq. (4). $|T|=\mathrm{n}$.

$C_{T}=\frac{1}{|T|} \sum_{j=1}^{|T|}\left(\frac{\left|{ }^{\bullet} t_{j}\right|}{\left|\bullet_{j}\right|+\left|t_{j}^{\bullet}\right|}\right)$ 
Both places and transitions correlation complexity of the model, $C_{r}$ as their average is in Eq. (5). Eq. (6) is the structural complexity degree.

$$
\begin{aligned}
& C r=\frac{C_{P}+C_{T}}{2} \\
& C_{s}=\lambda C_{n}+(1-\lambda) C_{r}
\end{aligned}
$$

Table 1 The structural complexity degree $\left(C_{s}\right)$.

\begin{tabular}{cc}
\hline The structural complexity score & Category \\
\hline 0 & Minimum \\
$0<C_{s}<\frac{1}{2}$ & Simple \\
$\frac{1}{2}$ & Ideal \\
$\frac{1}{2}<\mathrm{C}<1$ & Complex \\
1 & Maximum \\
\hline
\end{tabular}

The structural complexity of the system is at intervals of 0 to 1 , such as probability. The structural complexity score of a simple system approximates to 0 . The structural complexity of a complex system scores closer to 1 . An ideal system's structure complexity is approximately $\frac{1}{2}[15]$.

\section{The Norwegian traffic lights control system improvement}

The green light, yellow/amber light and red signals are the standard traffic lights states. One more stage in the Norwegian traffic lights, i.e., the red-yellow signal which lights up simultaneously. This redyellow signal is ahead of the red signal ends. The Norwegian traffic lights improvement change the redyellow signal of the Norwegian feature into a yellow signal only after the red signal ends. It referred to Vienna convention on road signs and signals in 1968. It is an additional state of the standard traffic lights used when the vehicles stream starting heavy traffic. In Norwegian traffic lights improvement, double yellow signals exist in a traffic lights cycle, i.e., ahead of the red signal ends and afterwards.

\section{Results and discussion}

This paper presents the model of the 2-phase traffic lights control system. The traffic lights control is integrated with the railway doorstop using the standard traffic lights, Norwegian and Norwegian improvement.

The structural complexity of a system has a positive correlation with the increase in the number of elements. The system is not appropriate to apply if the score approaches one while the elements of the system upgrades. The structural complexity increases if implement the multi-phase of the traffic lights. Does it mean that the system is unsuitable for actual implementation? It can solve by increasing the number of control systems on each element. A system with high complexity and abundant elements can return its complexity to an ideal system while establishing the coordination between components. It uses extra control systems. 
(a)

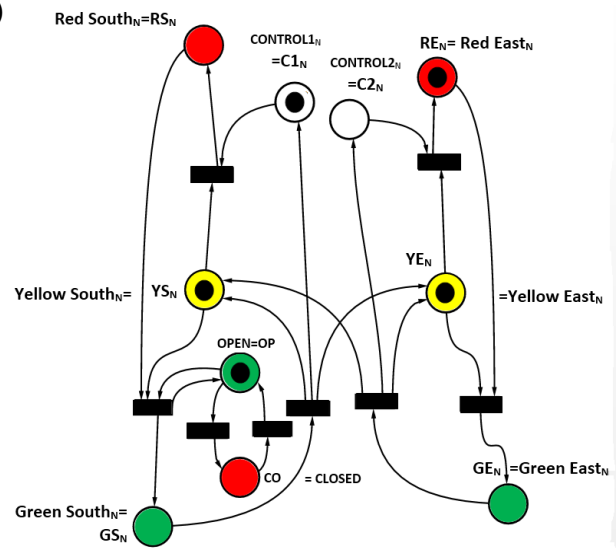

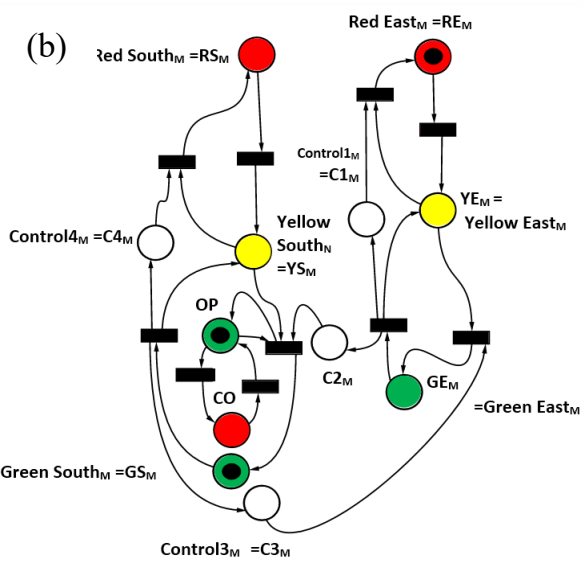

Figure 2 (a) The synchronized Norwegian traffic light model with the railway doorstop. (b) The synchronized Norwegian improvement with the railway doorstop.

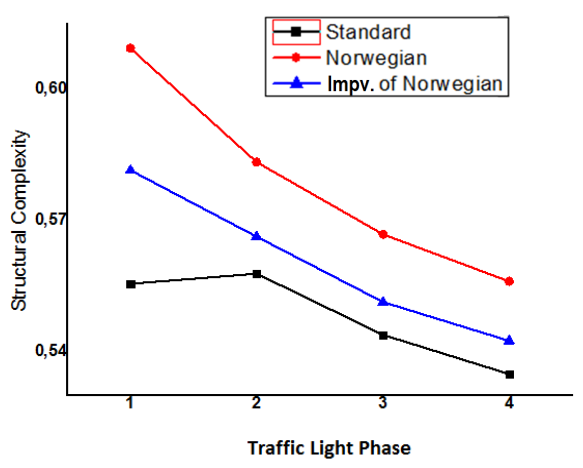

Figure 3 The regular traffic lights structural complexity has no additional feature.

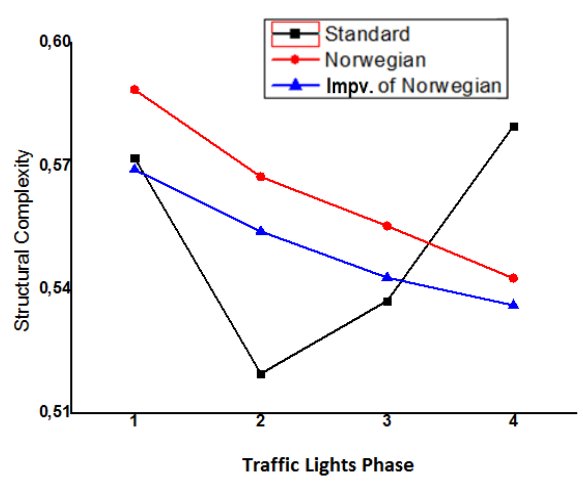

Figure 4 The structural complexity of synchronized traffic lights with the railway doorstop.

Figure 3 is the regular traffic lights add no feature. The structural complexity approximates to 0.5 or the ideal system when the number of phases increases. The standard traffic lights, Norwegian and Norwegian improvement are suitable for the massive use of the traffic lights phase.

The standard, the Norwegian and the Norwegian traffic lights improvement are appropriate for the actual implementation. The standard traffic light has the fewest transitions to trigger a previous state to be the next state. The transitions correspond to the number of controllers in an element of the system. The structural complexity will be high or complicated if the number of elements/states of a system increases. 
However, an increase in structural complexity does not occur when a control system accompanies each element.

Figure 4 is the traffic lights structural complexity that synchronized with the railway doorstop. The standard type is the best when implementing the 2-phase and 3-phase traffic lights. The Norwegian and Norwegian improvement have a steady downward trend when the traffic light phase increases. The Norwegian and Norwegian improvement structural complexities are better than the standard traffic lights that require adding controls while using the 4-phase traffic lights.

The synchronous system with the railway doorstop feature adds 2 states, namely CO and OP. It adds 2 transitions also. The state's number must have a balance to the rise in the number of the transition. It means that this feature must add at least dual controls. The Norwegian and the Norwegian traffic lights improvement have their control system over the standard system. $C 1_{N}$ and $C 2_{N}$ in Figure 2(a) and $C 1_{M}$, $\mathrm{C} 2{ }_{M}, C 3_{M}$ and $C 4_{M}$ in Figure 2(b) are the control places. Synchronous traffic lights with the railway doorstop features perpetuate the structural complexity trend of the Norwegian and its improvement. It always approximates to 0.5 when the phase of the traffic lights add.

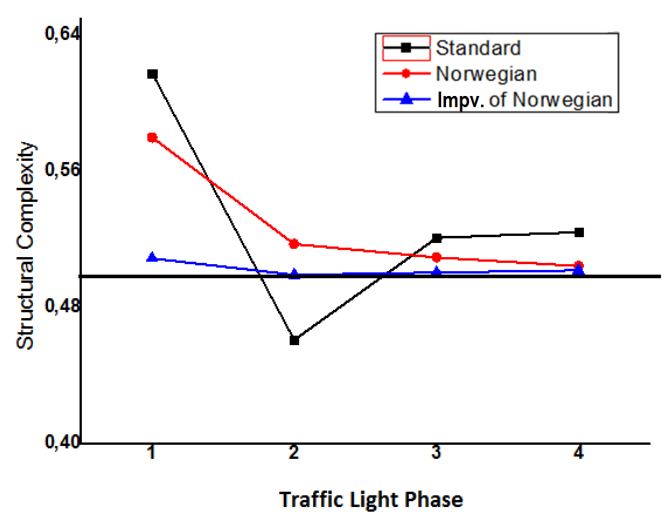

Figure 5 The structural complexity of the synchronized traffic lights to the railway doorstop and attached with extra green signal time intervals.

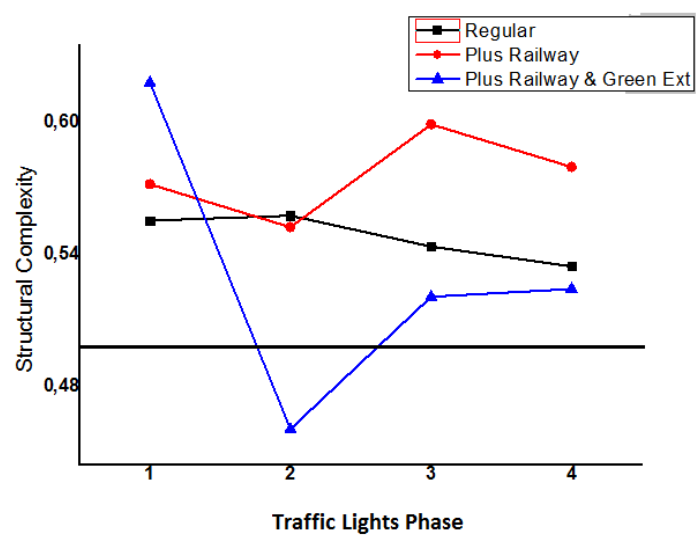

Figure 6 The standard traffic lights structural complexity adds using double features. 
It based on Figure 5. The traffic lights are synchronized to the railway doorstop and attached with extra green signal time intervals. The structural complexity of all types approaches 0.5. All types are suitable for use. The control systems of the extra green signal time intervals stabilize the system. The Norwegian traffic light improvement is best due to the own control for each element is the most. The structural complexity of the standard traffic lights fluctuate.

All trends of structural complexity approximate to 0.5 while the traffic lights are synchronized to the railway doorstop and uses the extra green signal time intervals. The structural complexity does not increase even though the number of features goes up. The system adds 3 places and 6 transitions in the feature for the extra green signal time intervals. The red ovoid in Figure 1 illustrates it. Implicitly, at least 6 control of the elements/transitions extra. The conclusion, adding several features can reduce the system structural complexity while extra controllers attached to it. Each system element can adequately function if under the supervision of a specific control system, although a system has a huge scale. The number of arcs overcomes the level of connectivity between elements of an extensive system. Structural complexity means the integration of a system with all existing elements.

Figure 6 is the standard traffic lights structural complexity that has added using double features. The standard traffic lights synchronized to a railway crossing have the worst structural complexity when it applies 3-phase and 4-phase traffic lights. The level of the structural complexity of the standard traffic lights, which synchronized to a railway crossing, tends to 0.5 while the number of phases continues to increase. The structural complexity of the standard traffic lights fluctuates while it is synchronized to a railway crossing and implements the extra green signal time interval.

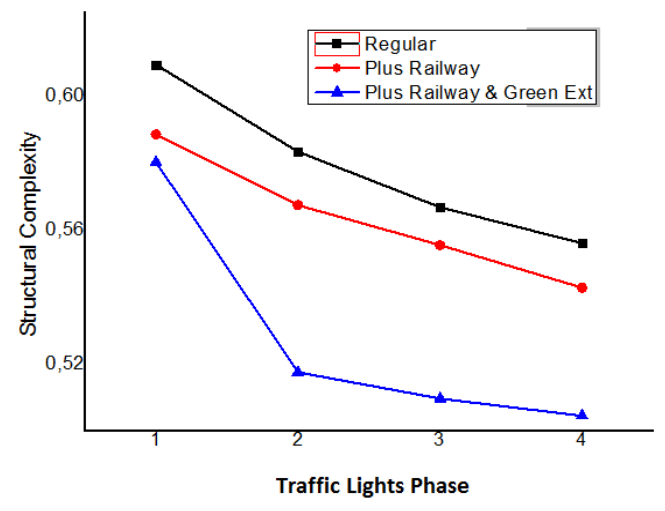

Figure 7 The structural complexity of the Norwegian traffic lights when synchronized with the railway doorstop and implements the extra green signal time intervals.

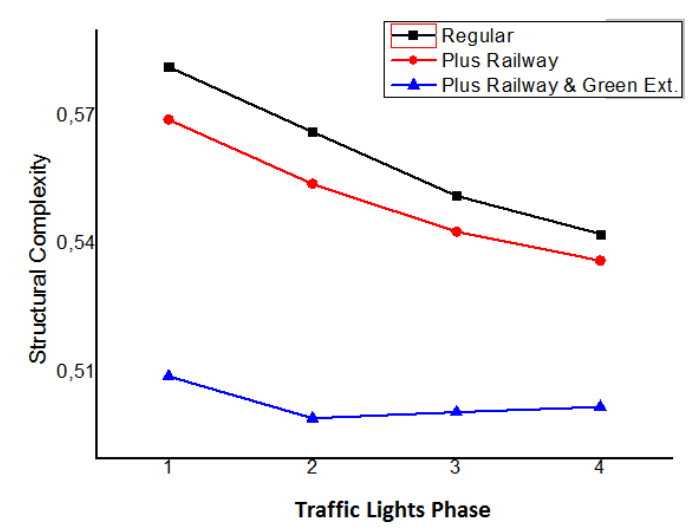

Figure 8 The structural complexity of the improvement of Norwegian traffic lights when synchronized with the railway doorstop and implements the extra green signal time intervals. 
Figure 7 notes that the Norwegian traffic lights' structural complexity is best when synchronized with the railway doorstop and implements the extra green signal time intervals. Figure 8 shows that the structural complexity of the improvement of Norwegian traffic lights. Their control system for green extension time intervals reduces the structural complexity, becoming an ideal system. All systems tend to be ideal structures due to the incremental traffic lights phase.

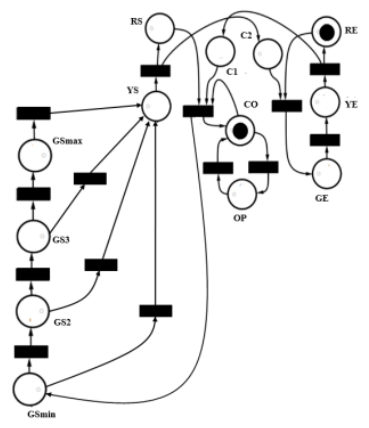

(a)

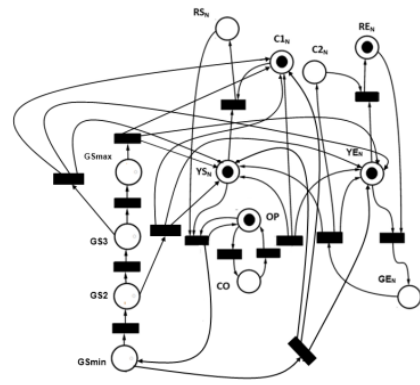

(b)

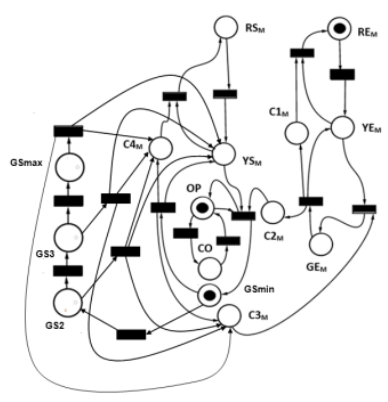

(c)

Figure 9 (a) Petri net model of standard traffic lights that have added dual feature. (b) Petri net model of the Norwegian traffic lights that have added dual feature. (c) Petri net model of the improvement of Norwegian traffic lights that have added dual features.

Based on Figures $\mathbf{7}$ and $\mathbf{8}$, both the Norwegian traffic lights and Norwegian improvement structural complexity have a steady downward trend approaching the ideal level. The system remains stable despite the features adds. Norwegian traffic light and its improvement controls for each element are the most. The standard traffic lights structural complexity fluctuates while features add. The standard system requires several control systems while synchronized to a railway doorstop and implements the extra green signal time interval.

Figure 9 is the structural complexity of the 2-phase traffic lights, which synchronized to the railway, plus the extra time interval for the green signals. The structural complexity of the standard traffic lights with the additional dual feature is 0.4775 , for the Norwegian traffic lights is 0.5176 . The structural complexity of Norwegian traffic lights improvement is 0.4995 . The Norwegian traffic lights structural complexities and Norwegian improvement are ideal. The complexity of standard types is simple. Figure 9 shows that the visualization of the structural complexities can be easily distinguished.

The Petri net connectivity of the traffic lights control systems can be represented using Forward Incidence and Backward Incidence matrices [27]. Furthermore, it can develop the algorithm of the structural complexity, and its analysis refers to the backward incidence matrix and the forward incidence matrix of the Petri net model.

Anyone can use the structural complexity algorithm attached to the Petri Net Toolbox. The required control system can be identified when it has determined the structural complexity of a system. The Toolbox can simulate, analyze and synthesize Discrete Event Systems based on Petri Net models.

\section{Conclusions}

It used the Petri net for determining the traffic light control system structural complexity. It added several features to the system, namely synchronized to the railway doorstop and the extra time interval of the green signal accompanied by the extra of a control system to each element. Thus, structural complexity becomes ideal for actual implementation. While the traffic lights integrated with the railway doorstop and had extra green signals time interval, the structural complexity of the Norwegian improvement system is the most ideal due to its design has the most number of controllers. The Norwegian traffic light is the runner up. The standard traffic lights are the lowest system. The Norwegian traffic light and its improvement are suitable for use on massive size systems. 


\section{Acknowledgements}

The Ministry of Education and Culture of Indonesia has funded this research in the vocational scheme.

\section{References}

[1] L Sihombing. The complexity of system. Praxis Framework, 2019

[2] AY Fridman. Planning and coordination in hierarchies of intelligent dynamic systems. Telkomnika 2016; 14, 1408-16.

[3] F Zhou, Y Su and X Fan. Low complexity sparse channel estimation based on compressed sensing. Telkomnika 2016; 14, 538-47.

[4] T Tristono, SD Cahyono, S Sudarno and P Utomo. Model of green extension traffic signal control system using Putri nets. Sendika 2019; 5, 84-92.

[5] CG Cassandras and S Lafortune. Introduction to discrete-event simulation. In: CG Cassandras and S Lafortune (Eds.). Springer, Massachusetts, 2008, p. 591-661.

[6] YS Huang and TH Chung. Modelling and analysis of traffic light control systems using timed colored Petri net. Intechopen, 2010, p. 567-90.

[7] T Murata. Petri net: Properties, analysis, and applications. Proc. IEEE 1989; 77, 541-80.

[8] G Popovicsa and L Monostoria. An approach to determine simulation model complexity. Proc. CIRP 2016; 52, 257-61.

[9] W Maraghy, H Maraghy, T Tomiyama and L Monostori. Complexity in engineering design and manufacturing. CIRP Ann. 2012; 61, 793-814.

[10] FR Hariri and JEW Perkasa. Measurement of e-learning system complexity using function-oriented metrics. Sem Nasional Inovasi Teknologi 2019; 3, 245-50.

[11] M Taiping and Z Peisi. Modeling \& performance analysis of manufacturing execution system based on Petri net. Open Cybern. Syst. J. 2015, 9, 1350-7.

[12] H Dong and X Li. Fault diagnosis for substation with redundant protection configuration based on time-sequence fuzzy Petri-net. Telkomnika 2013, 11, 231-40.

[13] I Mukhlash, WN Rumana, D Adzkiya and R Sarno. Business process improvement of production systems using coloured Petri nets. Bull. Electr. Eng. Informat. 2018; 7, 102-12.

[14] D Ouelhadj and S Petrovic. A survey of dynamic scheduling in the manufacturing system. J. Sched. $2009 ; 12,417-31$.

[15] D Han and Y Tian. Analysis and application of transition systems based on Petri nets and relation matrices to business process management. Math. Probl. Eng. 2020; 2020, 2545413.

[16] MDS Soares. 2010, Architecture-driven integration of modeling languages for the design of software-intensive systems. Master Thesis. Universidade Federal de Uberlândia, Uberlândia, Brazil.

[17] L Yang and Y Xiao-bo. The strategies of optimizing fuzzy Petri nets by using an improved genetic algorithm. Telkomnika 2016; 14, 62-8.

[18] D Hartanti, RN Aziza and PC Siswipraptini. Optimization of smart traffic lights to prevent traffic congestion using fuzzy logic. Telkomnka 2019; 17, 320-7.

[19] F Kurniawan, H Sajati and O Dinaryanto. Adaptive traffic controller based on pre-timed system. Telkomnika 2016; 14, 56-63.

[20] A Zinovyev and E Mirkesde. Data complexity measured by principal graphs. Comput. Math. Appl. 2013, 65, 1471-82.

[21] H Zenil, NA Kiani and J Tegnér. Review of graph and network complexity from an algorithmic information perspective. Entropy 2018; 20, 551.

[22] DL Neel and ME Orrison. The linear complexity of a graph. Electron. J. Combinator. 2006; 13, R9.

[23] RR Basir. Space and time complexity analysis on the growth of heap sort, insertion sort and merge algorithm with java programming. String 2020; 5, 109-18.

[24] WH Lee and CY Chiu. Design and implementation of a smart traffic signal control system for smart city applications. Sensors 2020; 20, 508 .

[25] D Adzkiya. 2008, Modeling traffic light using Petri net \& its simulation. Master Thesis. Institut Teknologi Sepuluh, Surabaya, Indonesia.

[26] IHCM. Direktorat bina marga direktorat bina jalan kota. IHCM, Jakarta, Indonesia, 1997.

[27] T Tristono, SD Cahyono, Sutomo and P Utomo. Investigate the complexity of the control system of the Norwegian traffic light using Petri net model. J. Phys. Conf. 2019; 1211, 012022. 